\title{
Analysis of Stability and Bifurcations of Limit Cycles in Chua's Circuit Through the Harmonic-Balance Approach
}

\author{
Fabrizio Bonani, Member, IEEE, and Marco Gilli, Member, IEEE
}

\begin{abstract}
This paper presents a spectral approach, based on the harmonic-balance technique, for detecting limit-cycle bifurcations in complex nonlinear circuits. The key step of the proposed approach is a method for a simple and effective computation of the Floquet multipliers (FM's) that yield stability and bifurcation conditions. As a case-study, a quite complex system, Chua's circuit, is considered. It is shown that the spectral approach is able to accurately evaluate the most significant bifurcation curves.
\end{abstract}

Index Terms - Bifurcation, Chua's circuit, frequency domain analysis, nonlinear circuits.

\section{INTRODUCTION}

$\mathbf{H}$ ARMONIC balance (HB) is a classical numerical technique for studying and designing electronic oscillators and nonlinear microwave circuits [1], [2]. In most applications, HB techniques have been used for determining the steady-state behavior of nonlinear circuits that exhibit one periodic attractor only. On the other hand, the global dynamics of nonlinear networks and systems are usually investigated through timedomain techniques that require the introduction of rather complex and sophisticated concepts [3].

Recently, some extensions of the HB technique have been proposed for the study of the global dynamic behavior and of bifurcation processes in nonlinear circuits that present several attractors. In [4] and [5] the authors have shown that the describing function technique (i.e., HB with a single harmonic) is able to predict the occurrence of chaotic behavior and several bifurcation phenomena.

The describing function technique presents the advantages of providing simple analytical results but, in general, it is not able to accurately predict all the complex dynamic phenomena occurring in nonlinear circuits, e.g., the various fold, flip, and homoclinic bifurcations of Chua's circuit, shown in [6]. To overcome this limitation, in [7]-[9] a method, based on the HB technique, for detecting fold and flip bifurcations was proposed. In [10] and [11] the local stability of limit cycles is analyzed through the application of Nyquist's theorem. In [12] bifurcations are studied through a spectral technique based on the introduction of measuring probes into the circuit. In [13] a spectral technique is exploited for studying flip

Manuscript received September 29, 1998; revised April 20, 1999. This paper was recommended by Associate Editor H. Kawakami.

The authors are with the Dipartimento di Elettronica, Politecnico di Torino, 10129 Torino, Italy.

Publisher Item Identifier S 1057-7122(99)06351-5. bifurcations in time-delayed systems. However, none of the above approaches provides a method for computing the limitcycle Floquet multipliers (FM's), which are the simplest tool for establishing the stability of the limit cycle and for detecting its bifurcations.

In [14, pp. 149-156] it is shown that the FM's can be determined to any desired accuracy by computing the eigenvalues of a finite matrix. This kind of computation is, however, not particularly suitable for imposing the fold and flip bifurcation conditions.

In this paper we propose a method for studying limitcycle bifurcations in nonlinear feedback systems based on the following steps.

1) Limit cycles are detected by using the HB technique as introduced in [15], which is fast and efficient even for a large number of harmonic.

2) The FM's are expressed as the roots of an algebraic equation of degree equal to the order of the system. Such an equation is derived through the extension to higher order systems of the technique proposed in [16] for second-order systems.

3) Stability and bifurcation conditions are easily established in terms of the coefficients of the above algebraic equation.

As a case-study we have considered Chua's circuit [17], [18], a rather complex dynamical system which exhibits a rich variety of dynamic behaviors, and we have shown that our method is able to accurately identify all its significant bifurcations.

The paper is structured as follows. In Section II the HB approach according to the formulation in [15] is briefly reviewed. In Section III, the technique for evaluating the FM's is presented. Finally, Section IV is devoted to the application of such techniques to the study of bifurcations in Chua's circuit.

\section{The HARMONIC-BALANCE APPROACH}

The harmonic-balance approach for detecting limit cycles in dynamical autonomous nonlinear systems can be briefly summarized as follows. Let us assume the nonlinear system can be expressed as a Lur'e system [4] (see Fig. 1)

$$
L(D) x(t)+n[x(t)]=s(t)
$$

where $D=d / d t$ is the time derivative, $L(\cdot)$ is a linear operator, $n(\cdot)$ is a nonlinear function, and $s(t)$ is a forcing 


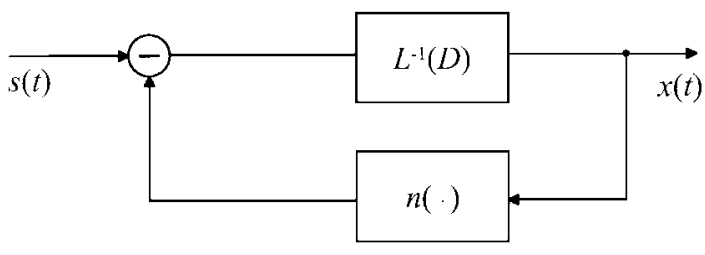

Fig. 1. Lur'e system.

term which is zero for autonomous systems. Both the linear operator and the nonlinear function can depend on several parameters. According to this assumption, the autonomous nonlinear system is defined by the dynamic equation

$$
L(D) x(t)+n[x(t)]=0 .
$$

Since the HB technique assumes a periodic solution takes place, $x(t)$ can be expanded as a superposition of harmonics (Fourier series)

$$
x(t)=\sum_{k=0}^{\infty} x_{k}(t)
$$

where

$$
\left\{\begin{array}{l}
x_{0}(t)=A_{0} \\
x_{k}(t)=A_{k} \cos (k \omega t)+B_{k} \sin (k \omega t) .
\end{array}\right.
$$

For computational purposes, the Fourier series has to be truncated to a suitable degree $N$, high enough to represent accurately the solution $x(t)$, thereby obtaining

$$
x(t)=\sum_{k=0}^{N} x_{k}(t)
$$

where the unknown variables to be determined through the HB technique are the $2 N+1$ spectral coefficients $A_{k}$ and $B_{k}$ and, at least for the autonomous case, the solution period $T=2 \pi / \omega$. Such $2 N+2$ independent equations are obtained [15] by sampling the dynamic equation (1) in $2 N+1$ time samples, uniformly spaced within the period-wide range $] 0, T]$

$$
t_{k}=\frac{T}{2 N+1} k, \quad k=1, \cdots, 2 N+1
$$

and imposing one of the coefficients of the fundamental harmonic to be zero. Without loss of generality, we shall assume hereafter $B_{1}=0$. The dynamic equation (1) is therefore converted in $2 N+2$ (nonlinear) algebraic equations involving the aforementioned unknown:

$$
\left\{\begin{array}{l}
\left.L(D) x(t)\right|_{t=t_{k}}+\left.n[x(t)]\right|_{t=t_{k}}=0 \\
B_{1}=0
\end{array}\right.
$$

where $k=1, \cdots, 2 N+1$.

Such a system can be efficiently assembled as follows. First, let us define the vectors of time samples $\underline{x}$ and spectral amplitudes $\underline{X}$

$\underline{x}=\left[x\left(t_{1}\right) \cdots x\left(t_{2 N+1}\right)\right]^{T}, \quad \underline{X}=\left[A_{0} A_{1} B_{1} \cdots A_{N} B_{N}\right]^{T}$.

By sampling (3) in the $2 N+1$ time samples (4), the two vectors are easily shown to be related by

$$
\underline{x}=\underline{\Gamma}^{-1} \underline{X} \Longleftrightarrow \underline{X}=\underline{\Gamma} \underline{x}
$$

where matrix $\underline{\underline{\Gamma}}^{-1}$

$$
\begin{aligned}
& \underline{\underline{\Gamma}}^{-1} \\
& =\left[\begin{array}{cccccc}
1 & \gamma_{1,1}^{c} & \gamma_{1,1}^{s} & \cdots & \gamma_{1, N}^{c} & \gamma_{1, N}^{s} \\
1 & \gamma_{2,1}^{c} & \gamma_{2,1}^{s} & \cdots & \gamma_{2, N}^{c} & \gamma_{2, N}^{s} \\
\vdots & \vdots & \vdots & & \vdots & \vdots \\
1 & \gamma_{2 N+1,1}^{c} & \gamma_{2 N+1,1}^{s} & \cdots & \gamma_{2 N+1, N}^{c} & \gamma_{2 N+1, N}^{s}
\end{array}\right]
\end{aligned}
$$

does not depend on the unknowns since $\gamma^{c}$ and $\gamma^{s}$ are given by

$$
\begin{aligned}
& \gamma_{p, q}^{c}=\cos \left(q \omega t_{p}\right)=\cos \left(\frac{q 2 \pi p}{2 N+1}\right) \\
& \gamma_{p, q}^{s}=\sin \left(q \omega t_{p}\right)=\sin \left(\frac{q 2 \pi p}{2 N+1}\right) .
\end{aligned}
$$

Furthermore, the application of the linear operator $L(D)$ to $x(t)$ [see (3)] can be described directly in terms of the harmonic components of $L(D) x(t)$ as the matrix product $\underline{\underline{\Omega}}(\omega) \underline{X}$ where

$$
\underline{\underline{\Omega}}(\omega)=\left[\begin{array}{cccccccc}
L(0) & 0 & 0 & 0 & 0 & \cdots & 0 & 0 \\
0 & R_{1} & I_{1} & 0 & 0 & \cdots & 0 & 0 \\
0 & -I_{1} & R_{1} & 0 & 0 & \cdots & 0 & 0 \\
0 & 0 & 0 & R_{2} & I_{2} & \cdots & 0 & 0 \\
0 & 0 & 0 & -I_{2} & R_{2} & \cdots & 0 & 0 \\
\vdots & \vdots & \vdots & \vdots & \vdots & & \vdots & \vdots \\
0 & 0 & 0 & 0 & 0 & \cdots & R_{N} & I_{N} \\
0 & 0 & 0 & 0 & 0 & \cdots & -I_{N} & R_{N}
\end{array}\right]
$$

and $R_{k}=\operatorname{Re}\{L(j k \omega)\}, I_{k}=\operatorname{Im}\{L(j k \omega)\}, k=1, \cdots, N$.

The algebraic system (5) is therefore expressed in the frequency domain as

$$
\left\{\begin{array}{l}
\underline{\Omega}(\omega) \underline{X}+\underline{N}\left(\underline{\underline{\Gamma}}^{-1} \underline{X}\right)=0 \\
B_{1}=0
\end{array}\right.
$$

where $\underline{N}\left(\underline{\underline{\Gamma}}^{-1} \underline{X}\right)=\underline{\underline{\Gamma}} \underline{n}\left(\underline{\underline{\Gamma}}^{-1} \underline{X}\right)$ and $\underline{n}\left(\underline{\underline{\Gamma}}^{-1} \underline{X}\right)$ denotes the vector of time samples of the nonlinear function $n(\cdot)$. Due to the term $N$, (9) is a nonlinear algebraic system in the $2 N+2$ unknowns $(\underline{X}, \omega)$ which can be efficiently solved by means of the Newton-Raphson technique [15] since the system Jacobian is

$$
\underline{\underline{J}}=\left[\begin{array}{cc}
\underline{\Omega}(\omega)+\frac{\partial \underline{\underline{N}}}{\partial \underline{X}} & \frac{\partial \underline{\underline{\Omega}}}{\partial \omega} \underline{X} \\
{\left[\begin{array}{lll}
0 & 0 & 1, \cdots, 0
\end{array}\right]} & 0
\end{array}\right]
$$

where the chain rule yields

$$
\frac{\partial \underline{N}}{\partial \underline{X}}=\underline{\Gamma} \frac{\partial \underline{n}}{\partial \underline{x}} \underline{\underline{\Gamma}}^{-1}
$$

and where $\partial \underline{n} / \partial \underline{x}$ is a diagonal matrix whose diagonal elements are simply the time samples of the derivative of the nonlinear function $n(\cdot)$. 


\section{Stability AND BifurCations of Limit Cycles}

As was pointed out in Section I, several approaches have been proposed for studying stability and bifurcations of limit cycles detected through the HB technique [4]-[13]. On the other hand, most time-domain methods for detecting bifurcations are based on the computation of the FM's since once the FM's are known, the bifurcation conditions are easily derived and the stability of the limit cycle is readily established as well. Unfortunately, the approaches proposed in [4]-[13] are not suitable for evaluating the FM's.

We shall show that for a Lur'e system, the FM's can be computed as the roots of an algebraic equation. Such an equation is obtained by extending to higher order systems the technique proposed in [16] for the evaluation of Hill's determinant (obtained from a second-order system). Then we shall point out that the conditions for fold and flip bifurcations can be expressed as simple constraints among the coefficients of the above equation.

As a first step, we consider a small perturbation $\tilde{x}(t)$ of the limit cycle $x(t)$ which must satisfy the variational equation

$$
L(D) \tilde{x}(t)+g(t) \tilde{x}(t)=0
$$

where

$$
g(t)=\left.\frac{d n(\zeta)}{d \zeta}\right|_{\zeta=x(t)}
$$

which describes a linear periodic time-varying system whose solution can be expressed as [19]

$$
\tilde{x}(t)=\sum_{i=1}^{M} H_{i} v_{i}(t) \exp \left(\lambda_{i} t\right)
$$

where $M$ is the order of the dynamical system, $H_{i}$ are suitable constants depending on the initial conditions, $v_{i}(t)$ are periodic functions of period $T$, and $\lambda_{i}$ are constant eigenvalues from which the FM's are easily determined as $\exp \left(\lambda_{i} T\right)$.

In order to determine the eigenvalues $\lambda_{i}$, we substitute the generic eigenfunction $v(t) \exp (\lambda t)$ into (11)

$$
L(D) v(t) \exp (\lambda t)+g(t) v(t) \exp (\lambda t)=0 .
$$

Notice that although $x(t)$ is represented in (3) by means of a finite number of harmonics, the periodic function $g(t)$ is, in general, expressed by an infinite number of harmonics, since it is the derivative of a nonlinear function evaluated in $x(t)$.
Therefore, both the periodic functions of period $T g(t)$ and $v(t)$ are expanded in a Fourier series as a superposition of exponential harmonics

$$
\begin{aligned}
& g(t)=\sum_{k=-\infty}^{\infty} G_{k} \exp (j k \omega t) \\
& v(t)=\sum_{k=-\infty}^{\infty} V_{k} \exp (j k \omega t) .
\end{aligned}
$$

By substituting (15) and (16) in (14) we have

$$
\begin{aligned}
& \sum_{k=-\infty}^{\infty} L(\lambda+j k \omega) V_{k} \exp (j k \omega t) \exp (\lambda t) \\
& =-\sum_{k=-\infty}^{\infty} G_{k} \exp (j k \omega t) \sum_{k=-\infty}^{\infty} V_{k} \exp (j k \omega t) \exp (\lambda t) .
\end{aligned}
$$

Then, equating the harmonic coefficients we obtain a linear homogeneous system whose unknowns are the harmonic components $V_{k}$. In order to have a nontrivial solution, the determinant $D(\lambda)$ of the infinite matrix $\underline{M}$ [see (18) at the bottom of this page] must vanish where $\tilde{\tilde{G}_{k}}=L(\lambda+j k \omega)+$ $G_{0}$. It can be verified that equation $D(\lambda)=0$ has infinitely many solutions of the type $\lambda_{i} \pm j k \omega(i=1, \cdots, M$ and $k=-\infty, \cdots, \infty)$ where $\lambda_{i}$ are the eigenvalues defined in (13).

For the sake of simplicity we restrict our attention to the case of a linear block $L(s)=P(s) / Q(s)$ with $\operatorname{deg}(P(s))=$ $\operatorname{deg}(Q(s))+1=M=3$, i.e., the case occurring in Chua's circuit.

Under this assumption, the term $L(\lambda)+G_{0}$ can be written as

$$
L(\lambda)+G_{0}=\eta \frac{\left(\lambda-\lambda_{s 1}\right)\left(\lambda-\lambda_{s 2}\right)\left(\lambda-\lambda_{s 3}\right)}{\left(\lambda-\lambda_{s 4}\right)\left(\lambda-\lambda_{s \tilde{s}}\right)}
$$

where $\eta$ is a suitable constant and $\left(\lambda_{s 1}, \lambda_{s 2}, \lambda_{s 3}\right),\left(\lambda_{s 4}, \lambda_{s \tilde{\nu}}\right)$ are zeros and poles, respectively, of $L(\lambda)+G_{0}$.

According to [16], in order to improve the convergence of the determinant $D(\lambda)$, it is convenient to introduce a new matrix $\underline{\underline{M}} 1=\operatorname{diag}\left\{f_{k}(\lambda)\right\} \underline{\underline{M}}$ with $f_{k}(\lambda)=[L(\lambda+j k \omega)+$ $\left.G_{0}\right]^{-1}$, shown in (20) at the bottom of this page, where $G_{q}^{(p)}(\lambda)=f_{p}(\lambda) G_{q}$.

$$
\begin{aligned}
& \underline{\underline{M}}=\left[\begin{array}{ccccccccccc}
\cdots & \cdots & \cdots & \cdots & \cdots & \cdots & \cdots & \cdots & \cdots & \cdots & \cdots \\
\cdots & G_{N} & \cdots & G_{1} & \tilde{G}_{-1} & G_{-1} & \cdots & G_{-N} & \cdots & \cdots & \cdots \\
\cdots & \cdots & G_{N} & \cdots & G_{1} & \tilde{G}_{0} & G_{-1} & \cdots & G_{-N} & \cdots & \cdots \\
\cdots & \cdots & \cdots & G_{N} & \cdots & G_{1} & \tilde{G}_{1} & G_{-1} & \cdots & G_{-N} & \cdots \\
\cdots & \cdots & \cdots & \cdots & \cdots & \cdots & \cdots & \cdots & \cdots & \cdots & \cdots
\end{array}\right] \\
& \underline{\underline{M}}_{1}=\left[\begin{array}{ccccccccc}
\cdots & \ldots & \ldots & \ldots & \ldots & \ldots & \ldots & \ldots & \ldots \\
G_{N}^{(-1)}(\lambda) & \ldots & G_{1}^{(-1)}(\lambda) & 1 & G_{-1}^{(-1)}(\lambda) & \ldots & G_{-N}^{(-1)}(\lambda) & \ldots & \ldots \\
\cdots & G_{N}^{(0)}(\lambda) & \ldots & G_{1}^{(0)}(\lambda) & 1 & G_{-1}^{(0)}(\lambda) & \ldots & G_{-N}^{(0)}(\lambda) & \ldots \\
\ldots & \cdots & G_{N}^{(1)}(\lambda) & \ldots & G_{1}^{(1)}(\lambda) & 1 & G_{-1}^{(1)}(\lambda) & \cdots & G_{-N}^{(1)}(\lambda) \\
\cdots & \cdots & \cdots & \cdots & \cdots & \ldots & \cdots & \cdots & \cdots
\end{array}\right]
\end{aligned}
$$


Denoting the determinant of $\underline{M}_{1}$ as $D_{1}(\lambda)$, by definition of $\underline{\underline{M}}$

$$
D(\lambda)=0 \Longleftrightarrow D_{1}(\lambda)=0 .
$$

The determinant $D_{1}(\lambda)$ exhibits infinitely many simple poles located in $\lambda_{s 1} \pm j k \omega, \lambda_{s 2} \pm j k \omega$, and $\lambda_{s 3} \pm j k \omega$. Moreover, due to the structure of $\underline{M}_{1}$, this determinant presents the same property of Hill's determinant [16], i.e., all poles located in $\lambda_{s i} \pm j k \omega i=1,2,3$ have the same residue.

Since one of the FM of a limit cycle is one (and, hence, one of the eigenvalues is zero) the determinant $D_{1}(\lambda)$ vanishes for $\lambda=0$. Therefore, the following Mittag-Leffler's expansion holds:

$$
\begin{aligned}
D_{1}(\lambda)= & c_{1} \sum_{k=-\infty}^{\infty}\left[\frac{1}{\lambda-\left(\lambda_{s 1}+j k \omega\right)}+\frac{1}{\left(\lambda_{s 1}+j k \omega\right)}\right] \\
& +c_{2} \sum_{k=-\infty}^{\infty}\left[\frac{1}{\lambda-\left(\lambda_{s 2}+j k \omega\right)}+\frac{1}{\left(\lambda_{s 2}+j k \omega\right)}\right] \\
& +c_{3} \sum_{k=-\infty}^{\infty}\left[\frac{1}{\lambda-\left(\lambda_{s 3}+j k \omega\right)}+\frac{1}{\left(\lambda_{s 3}+j k \omega\right)}\right] \\
= & \frac{j \pi}{\omega}\left[c_{1} \cot \left(\frac{j\left(\lambda-\lambda_{s 1}\right) \pi}{\omega}\right)+c_{1} \cot \left(\frac{j \lambda_{s 1} \pi}{\omega}\right)\right. \\
& +c_{2} \cot \left(\frac{j\left(\lambda-\lambda_{s 2}\right) \pi}{\omega}\right)+c_{2} \cot \left(\frac{j \lambda_{s 2} \pi}{\omega}\right) \\
& \left.+c_{3} \cot \left(\frac{j\left(\lambda-\lambda_{s 3}\right) \pi}{\omega}\right)+c_{3} \cot \left(\frac{j \lambda_{s 3} \pi}{\omega}\right)\right]
\end{aligned}
$$

where the three constants $c_{1}, c_{2}$, and $c_{3}$ are the residues of $D_{1}(\lambda)$ in $\lambda_{s 1}, \lambda_{s 2}$, and $\lambda_{s 3}$ and can be computed as the determinant of the matrices $\underline{\underline{R}}_{i}(i=1,2,3)$ as shown in (23) at the bottom of this page, where $\bar{G}_{q}=f_{0}^{i} G_{q}$ and

$$
\begin{aligned}
f_{0}^{1} & =\frac{\left(\lambda_{s 1}-\lambda_{s 4}\right)\left(\lambda_{s 1}-\lambda_{s 5}\right)}{\eta\left(\lambda_{s 1}-\lambda_{s 2}\right)\left(\lambda_{s 1}-\lambda_{s 3}\right)} \\
f_{0}^{2} & =\frac{\left(\lambda_{s 2}-\lambda_{s 4}\right)\left(\lambda_{s 2}-\lambda_{s \tilde{5}}\right)}{\eta\left(\lambda_{s 2}-\lambda_{s 1}\right)\left(\lambda_{s 2}-\lambda_{s 3}\right)} \\
f_{0}^{3} & =\frac{\left(\lambda_{s 3}-\lambda_{s 4}\right)\left(\lambda_{s 3}-\lambda_{s 5}\right)}{\eta\left(\lambda_{s 3}-\lambda_{s 1}\right)\left(\lambda_{s 3}-\lambda_{s 2}\right)} .
\end{aligned}
$$

From a computational standpoint, the residue $c_{i}$ is evaluated computing the determinant of matrix $\underline{\underline{R}}_{i}$, truncating the infinite representation of $g(t)$ to a finite number of harmonics $N$ which can be rather small since, owing to the structure of $\underline{R}_{i}$ and to the definition of $f_{k}(\lambda)$ given above, the infinite determinant of (23) is rapidly convergent (see [16, Sec. 2.8]). The spectral coefficients $G_{k}$ are determined through the relationship

$$
\underline{G}=\underline{\underline{\Gamma}} e \underline{g}
$$

where $\underline{G}=\left[G_{-N}, G_{-(N-1)}, \cdots, G_{0}, \cdots, G_{N-1}, G_{N}\right]^{T}, \underline{g}$ are the time samples of function $g(t)$

$$
\underline{g}=\left[g\left(t_{1}\right) \cdots g\left(t_{2 N+1}\right)\right]^{T}=g\left(\underline{\underline{\Gamma}}^{-1} \underline{X}\right)
$$

and

$$
\begin{gathered}
\underline{\underline{\Gamma}}_{e}^{-1}=\left[\begin{array}{ccccc}
\gamma_{-N,-N}^{e} & \cdots & \gamma_{-N, 0}^{e} & \cdots & \gamma_{(-N, N)}^{e} \\
\vdots & & \vdots & & \vdots \\
\gamma_{N,-N}^{e} & \cdots & \gamma_{N, 0}^{e} & \cdots & \gamma_{(N, N)}^{e}
\end{array}\right] \\
\gamma_{p, q}^{e}=\exp \left(j q \omega t_{p}\right)=\exp \left(\frac{j q 2 \pi p}{2 N+1}\right) .
\end{gathered}
$$

Moreover, since $\lim _{\operatorname{Re}[\lambda] \rightarrow \pm \infty} D_{1}(\lambda)=1$ and $\lim _{\operatorname{Re}[\lambda] \rightarrow \pm \infty} \cot (j \lambda)=\mp j$, the residues have to satisfy the following additional constraints:

$$
\begin{aligned}
& c_{1}+c_{2}+c_{3}=0 \\
& c_{1} \cot \left(\frac{j \lambda_{s 1} \pi}{\omega}\right)+c_{2} \cot \left(\frac{j \lambda_{s 2} \pi}{\omega}\right)+c_{3} \cot \left(\frac{j \lambda_{s 3} \pi}{\omega}\right) \\
& =\frac{\omega}{j \pi} .
\end{aligned}
$$

Therefore, the three residues can be efficiently determined by evaluating the determinant of only one of the three matrices $\underline{\underline{R}} i$.

Finally, substituting in (22)

$$
\mu=\exp (\lambda T)=\exp \left(\frac{2 \pi \lambda}{\omega}\right)
$$

exploiting (31) and taking into account that one of the FM's is one [see (22)], the other two FM's are determined as solutions of the following second-order algebraic equation:

$$
\mu^{2}+a \mu+b=0
$$

where

$$
\begin{aligned}
& a=-\frac{a_{N}}{a_{D}} \\
& a_{N}=c_{1} \mu_{s 1}\left(\mu_{s 2}-1\right)\left(\mu_{s 3}-1\right)\left(\mu_{s 2}+\mu_{s 3}\right) \\
& +c_{2} \mu_{s 2}\left(\mu_{s 1}-1\right)\left(\mu_{s 3}-1\right)\left(\mu_{s 1}+\mu_{s 3}\right) \\
& +c_{3} \mu_{s 3}\left(\mu_{s 1}-1\right)\left(\mu_{s 2}-1\right)\left(\mu_{s 1}+\mu_{s 2}\right) \\
& a_{D}=\mu_{s 1} \mu_{s 2} \mu_{s 3}\left[c_{1} \mu_{s 1}\left(\mu_{s 2}-1\right)\left(\mu_{s 3}-1\right)\right. \\
& +c_{2} \mu_{s 2}\left(\mu_{s 1}-1\right)\left(\mu_{s 3}-1\right) \\
& \left.+c_{3} \mu_{s 3}\left(\mu_{s 1}-1\right)\left(\mu_{s 2}-1\right)\right] \\
& b=\frac{1}{\mu_{s 1} \mu_{s 2} \mu_{s 3}} \\
& \mu_{s i}=\exp \left(-2 \lambda_{s i} \pi / \omega\right), \quad i=1,2,3 .
\end{aligned}
$$

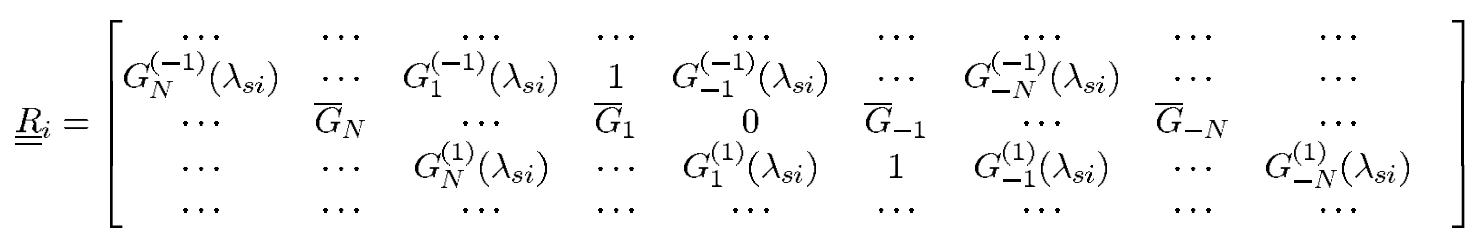




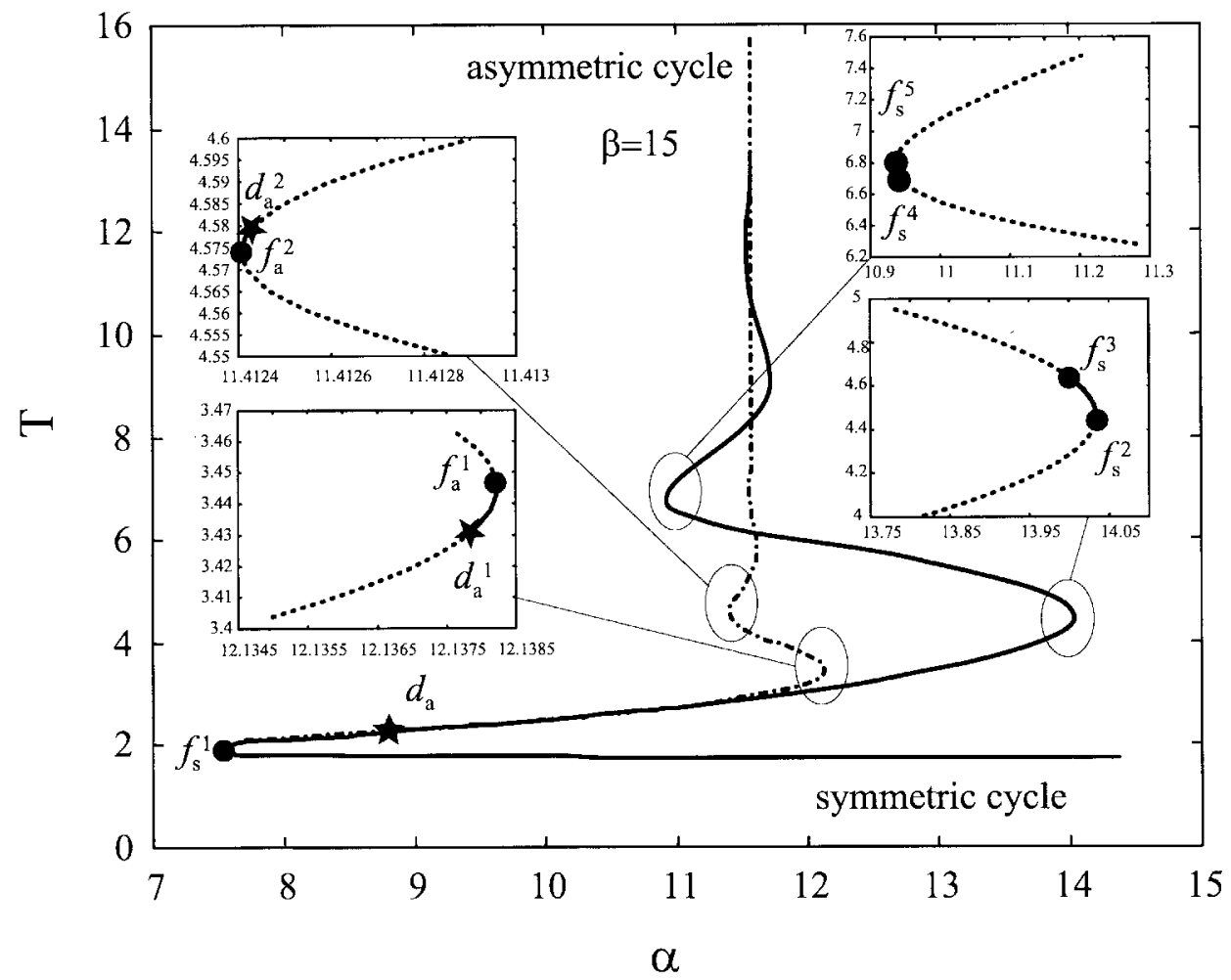

Fig. 2. Parameter $\alpha$ versus cycle period $T$ for both asymmetric and symmetric limit cycles. The HB system was solved with $N=21$ harmonics and $\beta=15$.

The conditions for fold and period doubling (flip) bifurcations are obtained by simply imposing that one FM equals one or -1 , respectively, i.e.,

$$
\begin{aligned}
\text { fold bifurcation } & \Longleftrightarrow 1+a+b=0 \\
\text { flip bifurcation } & \Longleftrightarrow 1-a+b=0 .
\end{aligned}
$$

We will show in the Section IV that by imposing that (9) and one of the conditions above are simultaneously satisfied, accurate bifurcation curves in the parameter space can be obtained.

\section{APPLICATION}

As an example of application, we shall analyze the bifurcation phenomena in a well-known dynamical system: the classical Chua's circuit [17], [18]. This choice is supported by the complex dynamic behavior of this system, thereby enabling a sound test for the HB approach to bifurcation analysis. The dynamic equations describing Chua's circuit can be written in terms of the normalized parameters $\alpha$ and $\beta$ as

$$
\begin{aligned}
& \frac{d x}{d t}=\alpha y-\alpha n(x)-\alpha x \\
& \frac{d y}{d t}=x-y+z \\
& \frac{z}{d t}=-\beta y .
\end{aligned}
$$

We assume that the memoryless nonlinear function $n(\cdot)$ can be approximated as a cubic nonlinearity [4]

$$
n(x)=-\frac{8}{7} x+\frac{4}{63} x^{3} .
$$

As shown in [4], such a system can be described as a Lur'e system, provided the following expression for the linear operator $L$ holds:

$$
L(D)=\frac{D^{3}+(1+\alpha) D^{2}+\beta D+\alpha \beta}{\alpha\left(D^{2}+D+\beta\right)} .
$$

Limit-cycle bifurcations of a Chua's circuit with smooth nonlinearity have been thoroughly investigated in [6], through theoretical and time-domain numerical techniques. In order to validate the $\mathrm{HB}$ approach, we shall address the most significant bifurcations reported in [6] and we shall show they can be accurately and efficiently detected by spectral techniques. It is possible to show that Chua's circuit, as described by the aforementioned functions exhibits, for $\alpha<7$, two stable equilibria symmetric with respect to the origin of the state space $(x, y, z)$. For $\alpha=7$, a Hopf bifurcation gives rise to two asymmetric limit cycles, which in turn are symmetric with respect to the origin since the nonlinear system (40) is odd. By further increasing $\alpha$, a fold bifurcation occurs, yielding a pair of symmetric limit cycles (one stable and one unstable). We start our investigation for values of $\alpha$ and $\beta$ (e.g., $\alpha=8$ and $\beta=15)$ for which the two stable asymmetric limit cycles and the stable symmetric limit cycle coexist (see [6, Sec. 3.2]). By solving the HB system for $\alpha=8$ and $\beta=15$, the asymmetric and the symmetric limit cycles have been detected. For $\beta$ held fixed, both cycles have then been continued with respect to the cycle period $T$. Therefore, the nonlinear algebraic system (9) is solved with $X$ and $\alpha$ as unknowns. The values of $\alpha$ versus the cycle period $T$ for $\beta=15$ are shown in Fig. 2, which has been obtained with $N=21$ harmonics (including dc).

For both symmetric and asymmetric limit cycles and for each value of $\alpha$ and $T$, the two FM's not identically equal to one have been evaluated through (33). In both cases, one FM always has a magnitude less than one. The other FM 


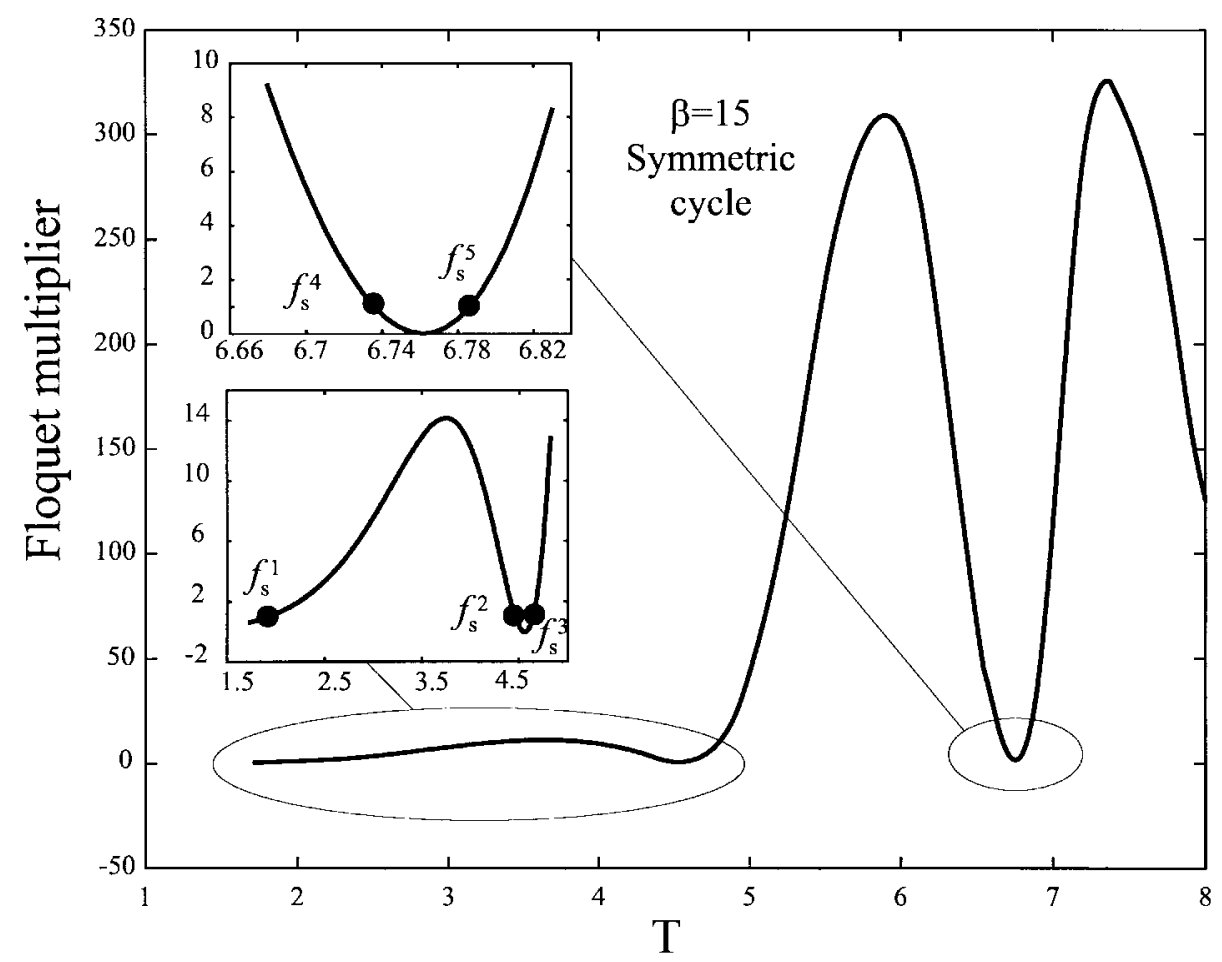

Fig. 3. Principal FM as a function of $T$ for the symmetric limit cycle and $\beta=15$.

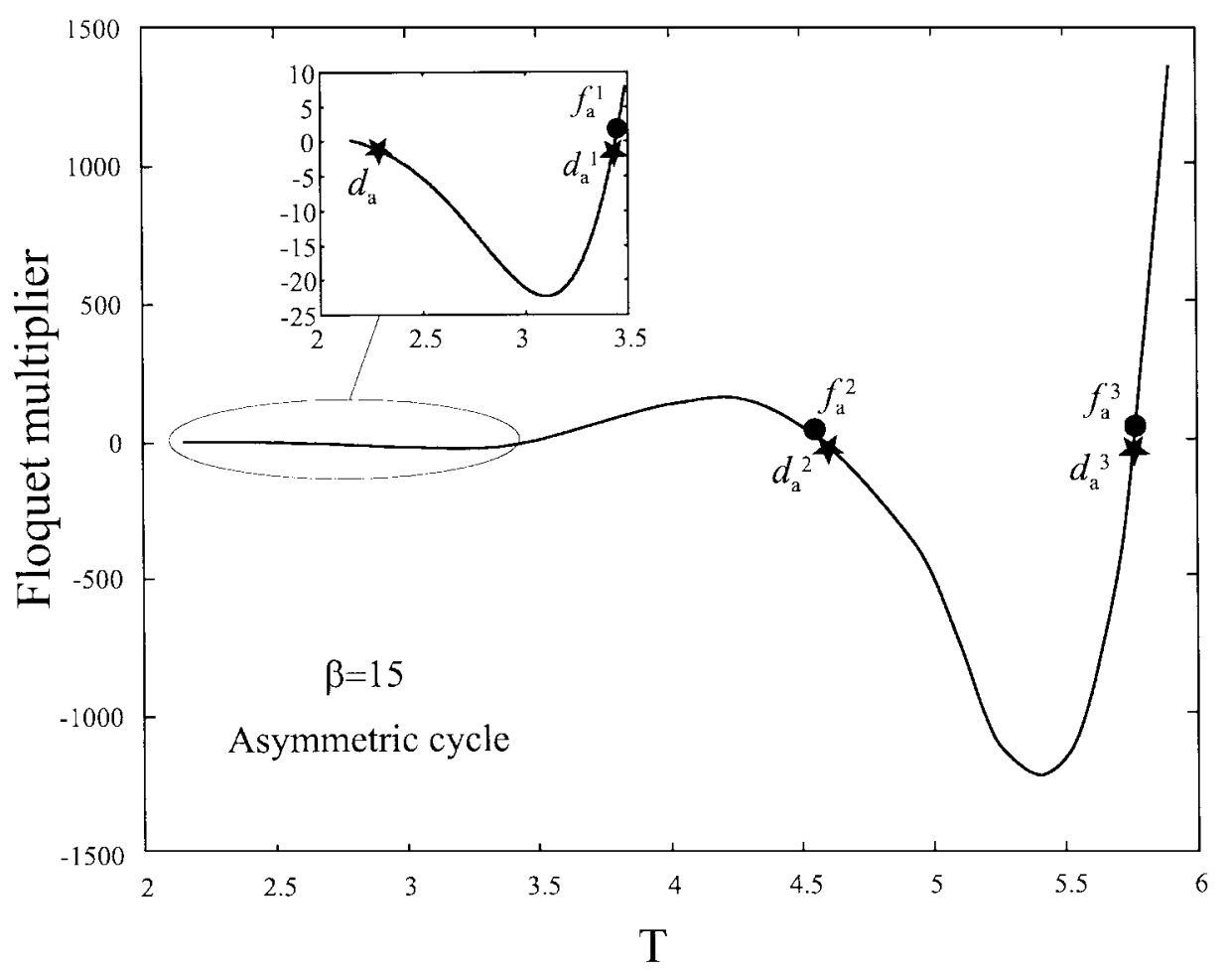

Fig. 4. Principal Floquet multiplier as a function of $T$ for the asymmetric limit cycle and $\beta=15$.

(hereinafter called the principal FM) is real and is responsible for all the limit-cycle bifurcations.

The principal FM's are reported as a function of period $T$ and for $\beta=15$ in Figs. 3 and 4 for the symmetric and the asymmetric limit cycles, respectively.

As far as the symmetric limit cycle is concerned (Fig. 3), the following bifurcations can be detected.
- A fold bifurcation (denoted as $f_{s}^{1}$ ) corresponding to the period $T$ for which the principal FM first takes the value +1 . The bifurcation $f_{s}^{1}$ gives rise to a pair of symmetric limit cycles, one unstable and one stable.

- As period $T$ is increased, the unstable symmetric limit cycle undergoes a sequence of fold bifurcations for those values of $T$ corresponding to a principal FM equal to 


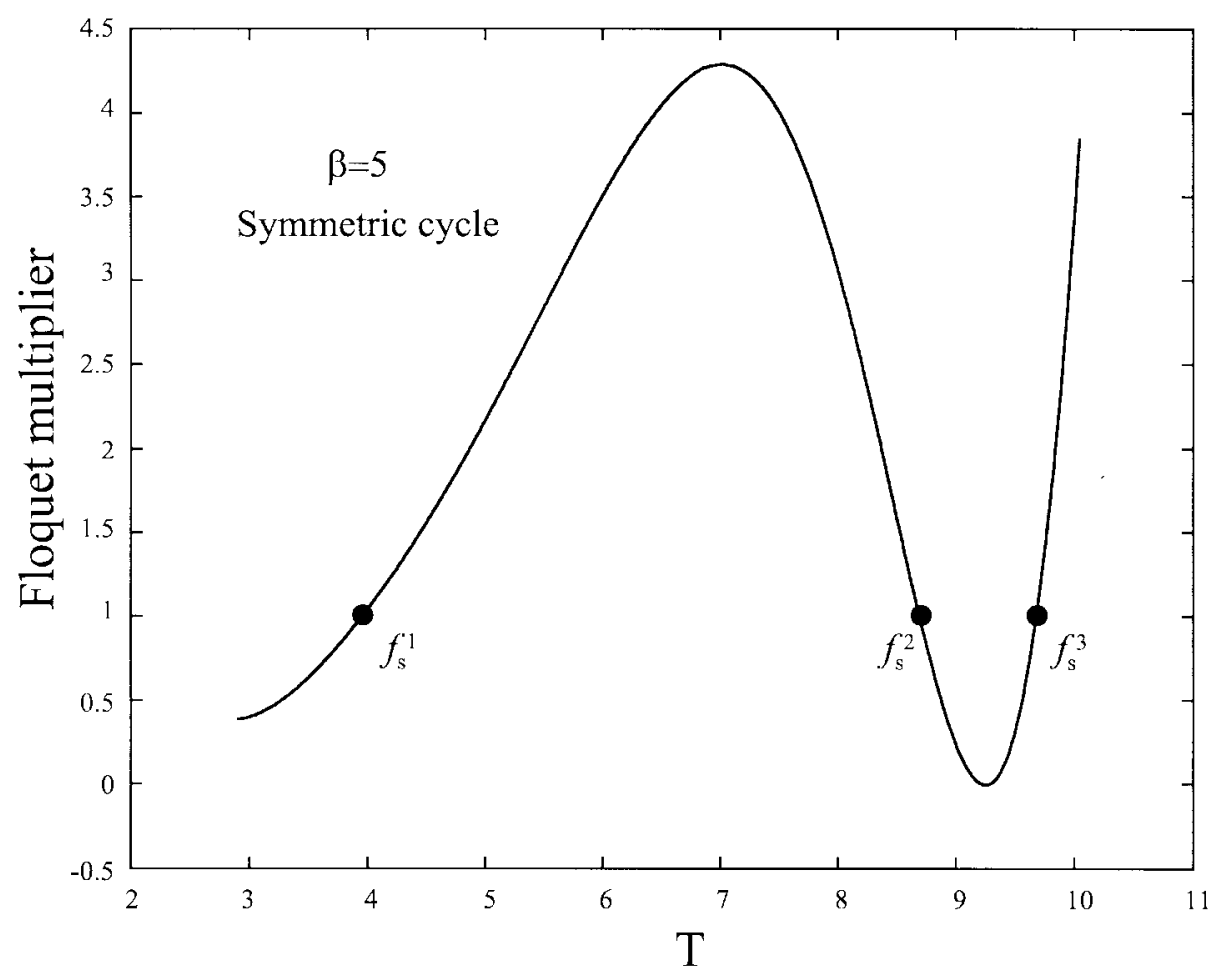

Fig. 5. Principal FM as a function of $T$ for the symmetric limit cycle and $\beta=5$.

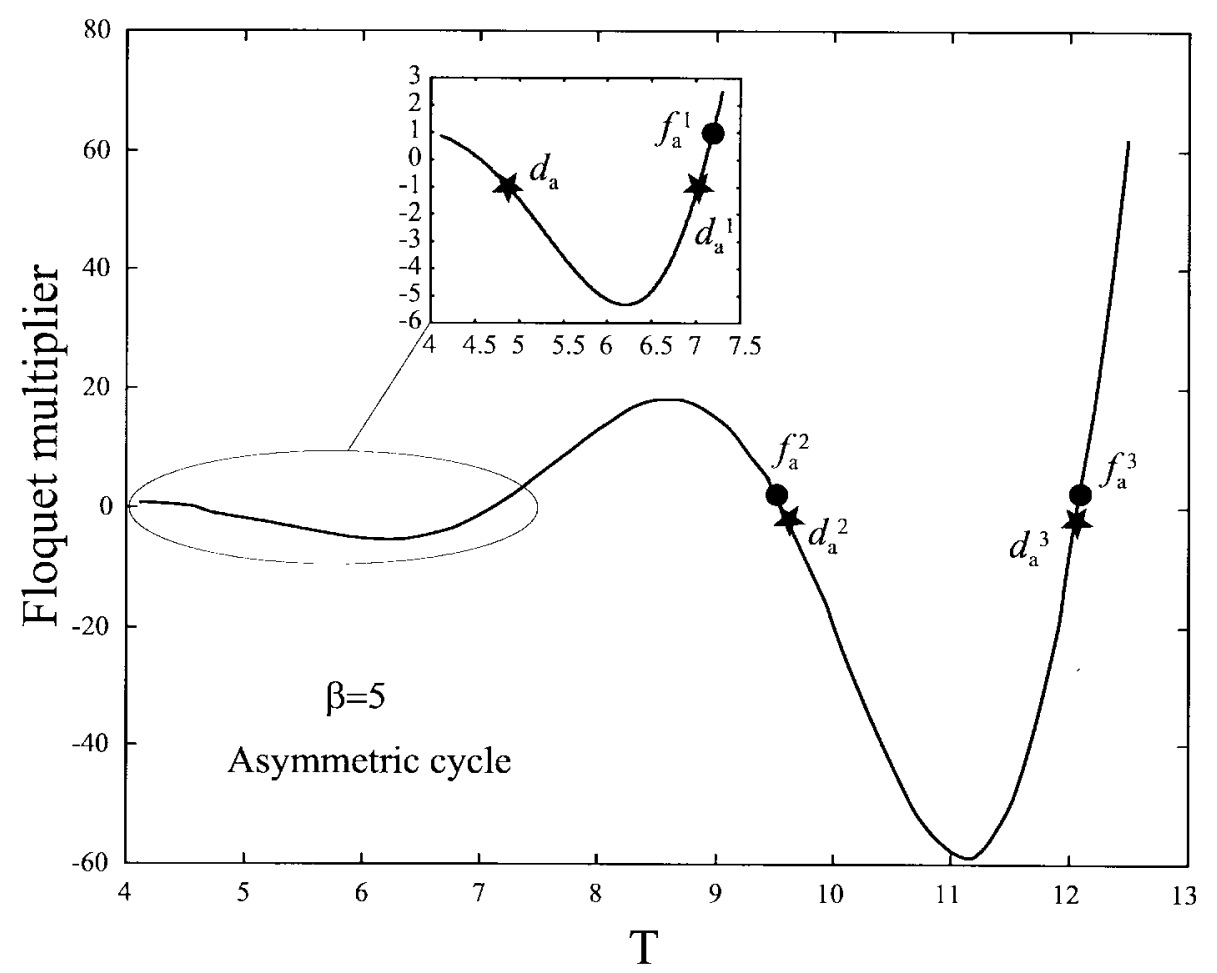

Fig. 6. Principal FM as a function of $T$ for the asymmetric limit cycle and $\beta=5$.

+1 . The first four such bifurcations (indicated as $f_{s}^{i}, i=$ $2,3,4,5)$ are shown in Fig. 3.

Notice that: 1) the fold bifurcations $f_{s}^{i}, i=1,2,5$ arise for values of $\alpha$ corresponding to a vertical tangent of the $T(\alpha)$ curve of the symmetric limit cycle (solid curve of Fig. 2); 2) the symmetric limit cycle does not present flip bifurcations because the principal FM is always greater or equal to zero (and the module of the other FM is always less than one); and 3) the symmetric limit cycle is stable for values of $T$ occurring before the bifurcation $f_{s}^{1}$ and within bifurcations $f_{s}^{2}-f_{s}^{3}, f_{s}^{4}-f_{s}^{5}$ (because, as shown in Fig. 3, the principal FM lies in the interval ]0, 1[).

With respect to the asymmetric limit cycle (Fig. 4), the following bifurcations are revealed. 




Fig. 7. Principal FM as a function of $T$ for the symmetric limit cycle and $\beta=25$.

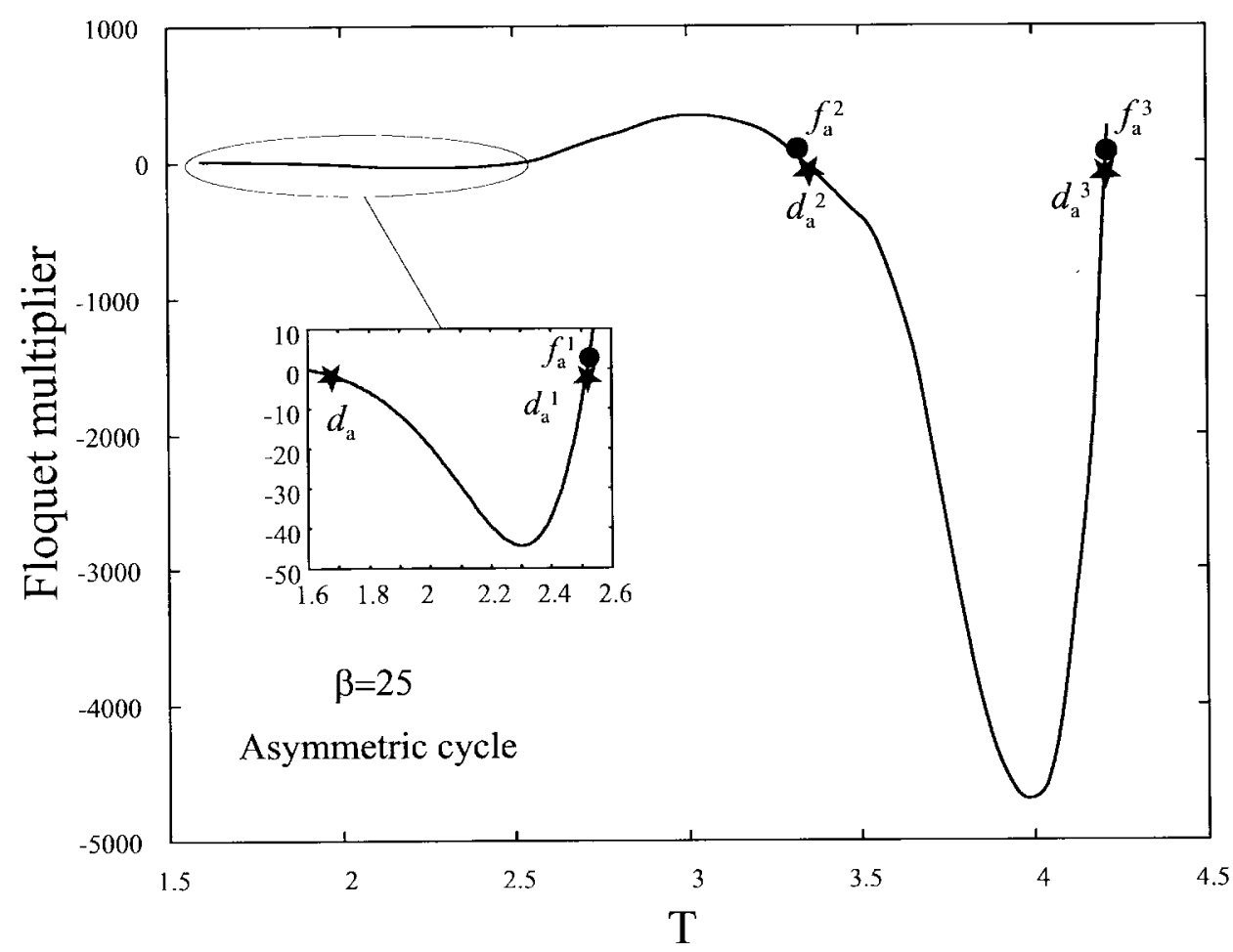

Fig. 8. Principal Floquet multiplier as a function of $T$ for the asymmetric limit cycle and $\beta=25$.

- The period-doubling bifurcation $d_{a}$, giving rise to the well-known spiral attractor that occurs for a period $T$ corresponding to the first crossing of value -1 by the principal FM.

- A sequence of flip and fold bifurcations. The first six such bifurcations: $d_{a}^{1}-f_{a}^{1}-f_{a}^{2}-d_{a}^{2}-d_{a}^{3}-f_{a}^{3}$ are shown in Fig. 4. Notice that $d_{a}^{i}(i=1,2,3)$ are flip bifurcations for which the principal FM equals $-1 . f_{a}^{i}(i=1,2,3)$ are fold bifurcations corresponding to a principal FM equal to +1 .

We remark that: 1) the asymmetric limit cycle is stable for values of $T$ such that the principal FM lies in the interval ]-1, 1[, i.e., before the bifurcation $d_{a}$ and within the bifurcations $d_{a}^{1}-f_{a}^{1}, f_{a}^{2}-d_{a}^{2}, d_{a}^{3}-f_{a}^{3}$ and 2) the fold bifurcations 


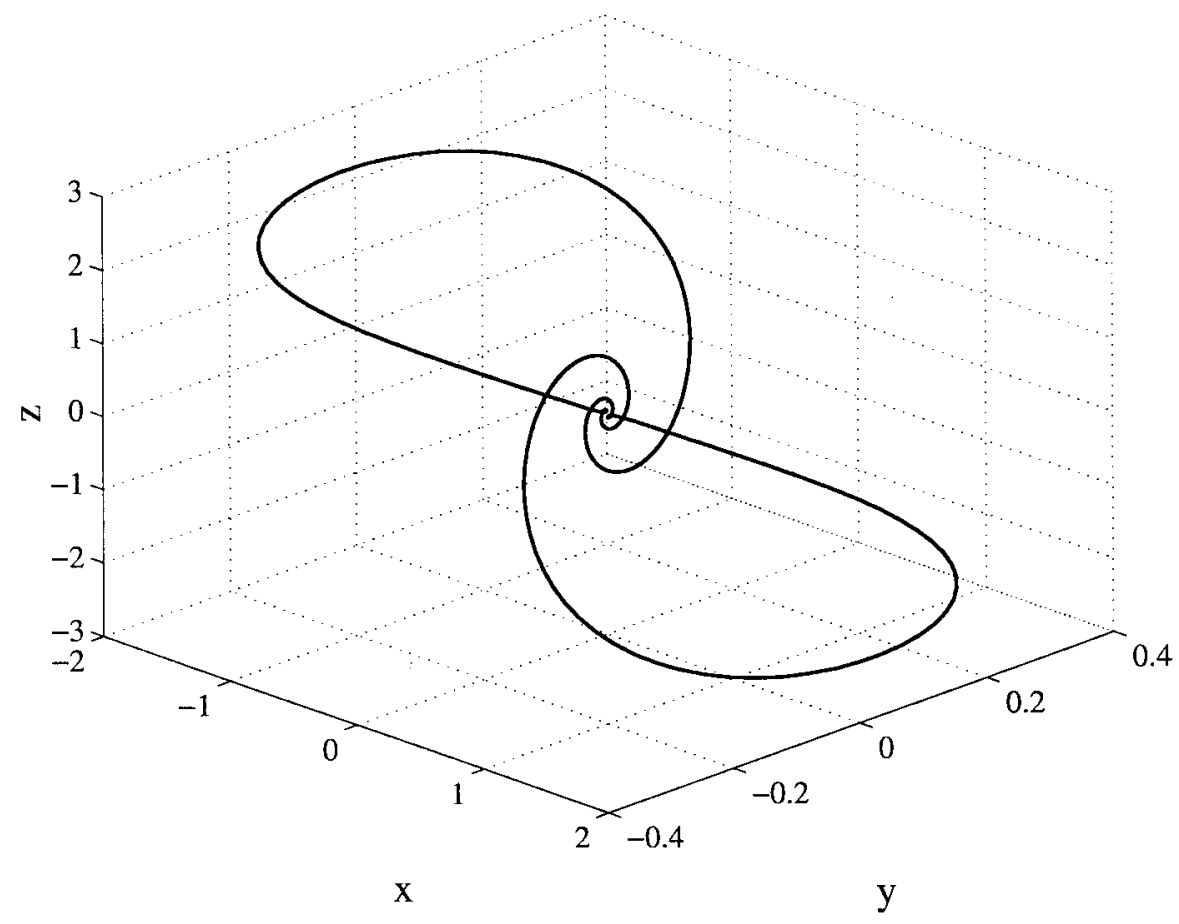

Fig. 9. Periodic orbit obtained from the symmetric limit cycle through the HB system for $T=13.3685, \alpha=11.5875, \beta=15$. The cycle is very close to the homoclinic loop lying around the origin of the state space. The HB system was solved with $N=21$ harmonics.

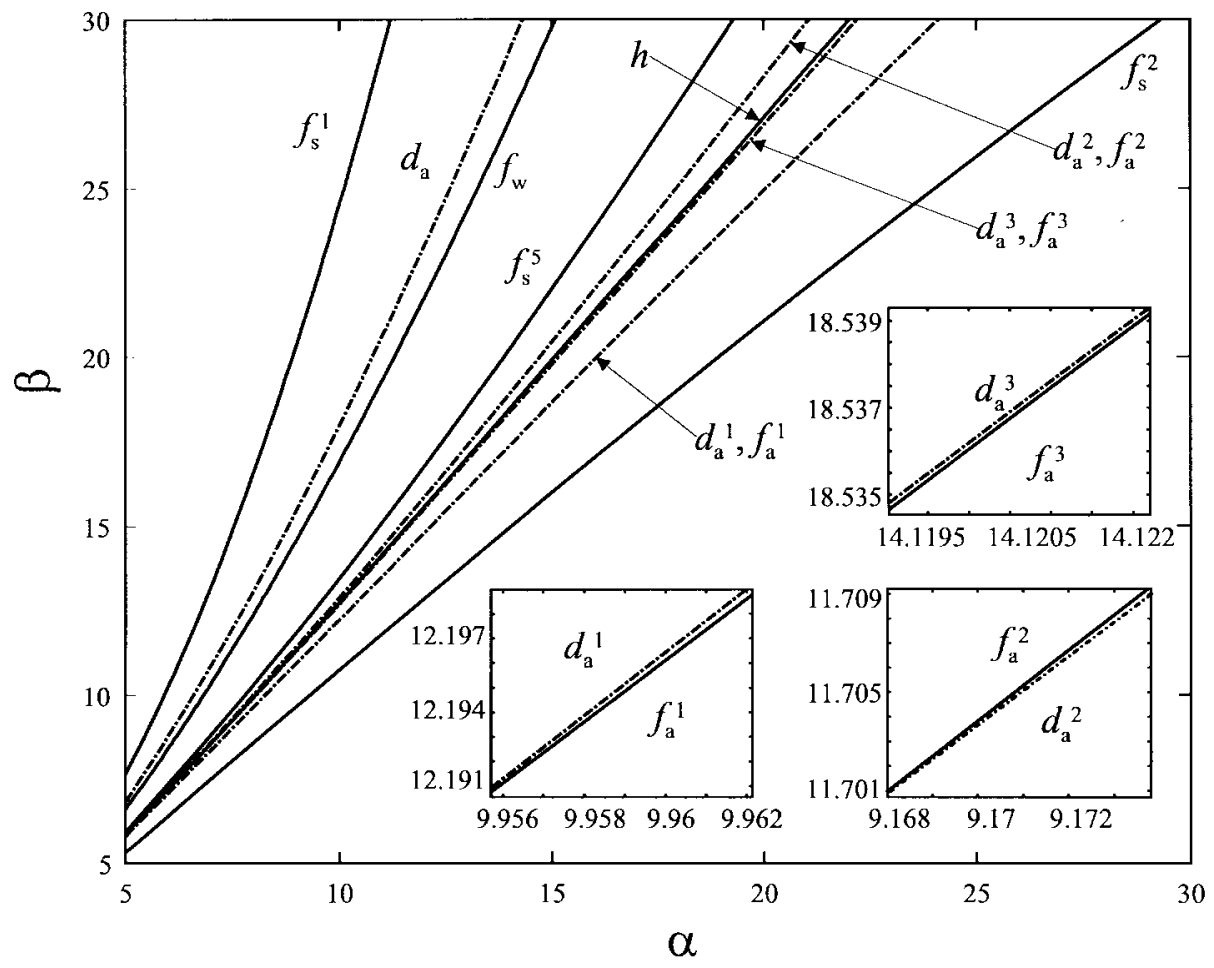

Fig. 10. Bifurcation curves in the parameter space.

$f_{a}^{i}(i=1,2,3)$ occur for values of $\alpha$ corresponding to a vertical tangent of the asymmetric limit cycle $T(\alpha)$ curve (dashed curve in Fig. 2).

The principal FM's are also shown in Figs. 5-8 for $\beta=5$ and $\beta=25$, respectively. It is worth noting that the same fold and flip bifurcations of the case $\beta=15$ can be observed.

Fig. 2 also yields a strong indication of the existence of a homoclinic orbit, due to the fact that both curves exhibit a vertical asymptote. In order to verify this conjecture, we have plotted the symmetric limit cycle obtained through the HB system for $T=13.3685, \alpha=11.5875, \beta=15$ in Fig. 9. The resulting orbit appears to be very close to the homoclinic loop arising from the intersection between the stable and unstable manifolds of the origin of the phase space.

Starting from the bifurcation points detected from Figs. 3 and 4 , bifurcation curves have been obtained by continuation 
with respect to $\beta$. The bifurcations have been evaluated by solving (9) and condition (39) with $\underline{X}, T$ and $\alpha$ as unknowns. We have also detected the fold bifurcation $f_{w}$ which gives rise to the period-three orbit window lying within the chaotic spiral attractor region of the parameter space. Finally, the homoclinic bifurcation $h$ has been evaluated as the vertical asymptote of the $T(\alpha)$ curve for several values of $\beta$. The most significant bifurcation curves in the parameter space are reported in Fig. 10. Since curves $d_{a}^{i}-f_{a}^{i}(i=1,2,3)$ are very close to each other, the insets in Fig. 10 permit us to distinguish between them. It is seen that, for a given value of $\beta$, by increasing $\alpha$ one encounters the curves corresponding to the following sequence of bifurcations: $f_{s}^{1}-d_{a}-f_{w}-f_{s}^{5}-$ $f_{a}^{2}-d_{a}^{2}-h-d_{a}^{3}-f_{a}^{3}-d_{a}^{1}-f_{a}^{1}-f_{s}^{2}$. This is in agreement with the relative positions of the bifurcation points shown in Fig. 2.

\section{CONCLUSION}

We have presented a spectral approach, based on the harmonic-balance technique, for the analysis of stability and limit-cycle bifurcations in complex nonlinear systems.

The approach is based on the following steps. 1) limit cycles are detected by using the HB technique proposed in [15], which allows the use of a large number of harmonics with a low computational burden; 2) by extending the technique shown in [16], the FM's of each limit cycle are computed as the roots of a low-order algebraic equation; and 3) then bifurcation conditions are expressed in terms of the coefficients of this equation.

The method was applied to Chua's circuit, since this complex dynamical system exhibits a large number of attractors and bifurcation processes. We have shown that, through the proposed spectral approach, a detailed analysis of limit-cycle stability is feasible. Moreover fold, flip, and homoclinic bifurcation curves have been accurately evaluated.

We remark that the above spectral technique is also suitable for studying circuits dispersive in time and distributed systems that admit of a simple description only in the frequency domain.

\section{REFERENCES}

[1] K. S. Kundert and A. Sangiovanni-Vincentelli, "Simulation of nonlinear circuits in the frequency domain," IEEE Trans. Computer-Aided Design, pp. 521-535, Oct. 1986 .

[2] A. Ushida, T. Adachi, and L. O. Chua, "Steady-state analysis of nonlinear circuits, based on hybrid methods," IEEE Trans. Circuits Syst. I, vol. 39, pp. 649-661, Aug. 1992.

[3] Y. A. Kuznetov, Elements of Applied Bifurcation Theory. New York: Springer-Verlag, 1995.

[4] R. Genesio and A. Tesi, "A harmonic balance approach for chaos prediction: Chua's circuit," Int. J. Bifurcation Chaos, vol. 2, no. 1, pp. 61-79, 1992.

[5] M. Basso, R. Genesio, and A. Tesi, "A frequency method for predicting limit cycle bifurcations," unpublished.

[6] A. I. Khibnik, D. Roose, and L. O. Chua, "On periodic orbits and homoclinic bifurcations in Chua's circuit with a smooth nonlinearity," J. Circuits, Syst. Comput., pp. 145-178, 1993.

[7] C. Piccardi, "Bifurcations of limit cycles in periodically forced nonlinear systems: The harmonic balance approach," IEEE Trans. Circuits Syst. I, vol. 41, pp. 315-320, Apr. 1994.
[8] C. Piccardi, "Bifurcation analysis via harmonic balance in periodic systems with feedback structure," Int. J. Control, vol. 62, pp. 1507-1515, 1995.

[9] _ "Harmonic balance analysis of codimension-2 bifurcations in periodic systems," IEEE Trans. Circuits Syst., vol. 43, pp. 1015-1018, Dec. 1996.

[10] V. Rizzoli and A. Neri, "State of the art and present trends in nonlinear microwave techniques," IEEE Trans. Microwave Theory Tech., pp. 343-365, Feb. 1988

[11] V. Rizzoli, A. Neri, and D. Masotti, "Local stability analysis of microwave oscillators based on Nyquist's theorem," IEEE Microwave Guided Wave Lett., vol. 7, pp. 341-343, Oct. 1998.

[12] A. Suarez, J. Morales, and R. Quéré, "Synchronization analysis of autonomous microwave circuits using new global-stability analysis tools," IEEE Trans. Microwave Theory Tech., vol. 46, pp. 494-504, May 1998.

[13] D. W. Berns, J. L. Moiola, and G. Chen, "Predicting period-doubling bifurcations and multiple oscillations in nonlinear time-delayed feedback systems," IEEE Trans. Circuits Syst. I, vol. 45, pp. 759-763, July 1998.

[14] A. I. Mees, Dynamics of Feedback Systems. New York: Wiley, 1981.

[15] K. S. Kundert, A. Sangiovanni-Vincentelli, and J. K. White, SteadyState Methods for Simulating Analog and Microwave Circuits. Boston, MA: Kluwer, 1990.

[16] E. T. Whittaker and G. N. Watson, A Course on Modern Analysis, 4th ed. Cambridge, U.K.: Cambridge Univ. Press, 1996, pp. 413-417.

[17] R. N. Madan, Ed., "Special issue on Chua's circuit: A paradigm for chaos," J. Circuits, Syst. Comput., vol. 3, Mar. 1993.

[18] _ "Special issue on Chua's circuit: A paradigm for chaos," $J$ Circuits, Syst. Comput., vol. 3, June 1993.

[19] F. M. Callier and C. A. Desoer, Linear System Theory. Heidelberg, Germany: Springer-Verlag, pp. 51-54, 1991.



Fabrizio Bonani (S'89-M'96) was born in Torino, Italy, in 1967. He received the Laurea degree, (cum laude) and the Ph.D. degree in electronic engineering from the Politecnico di Torino, Torino, Italy, in 1992 and 1996, respectively.

Since 1995, he has been a Researcher at the Electronics Department, Politecnico di Torino. His research interests are mainly in the areas of the physics-based simulation of semiconductor devices, with special emphasis on the noise analysis of microwave field-effect and bipolar transistors, and the thermal analysis of power microwave circuits. Part of his research concerns the analysis and simulation of nonlinear dynamical systems. From October 1994 to June 1995, he was with the ULSI Technology Research Department, Bell Laboratories, Murray Hill, NJ, as a Consultant, working on physicsbased noise modeling of electron devices.

Dr. Bonani is is member of the Associazione Elettrotecnica Italiana (AEI).

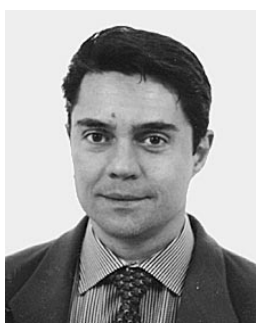

Marco Gilli (M'92) received the Dr. Eng. degree in electronics engineering from the Politecnico di Torino, Torino, Italy, in 1989.

From November 1991 to November 1998, he was a Researcher (Assistant Professor) at the Department of Electronics, Politecnico di Torino. Since November 1998 he has been an Associate Professor of electrical engineering at the Faculty of Engineering, Politecnico di Torino. His research activity is mainly in the area of nonlinear circuits and systems and neural networks and partially in the field of electromagnetic compatibility.

Dr. Gilli received the Best Paper Award from the International Journal of Circuit Theory and Applications in 1994 and in 1998, he was recipient of the Ravani Award of the Academy of Science of Turin, for his research in electrical engineering. 